\title{
Original
}

\section{Effects of an in Vitro Reconstructed Three-dimensional Hematopoietic Microenvironment on Bone Regeneration in a Rat Calvarial Defect Model}

\author{
Shingo Hirashima ${ }^{1,2}$, Keisuke Ohta ${ }^{1,3)}$, Masahiko Hagihara ${ }^{4)}$, Motohisa Shimizu ${ }^{4)}$, Tomonoshin Kanazawa ${ }^{1)}$ and Kei-ichiro Nakamura ${ }^{1)}$ \\ 1) Division of Microscopic and Developmental Anatomy, Department of Anatomy, Kurume University School of Medicine, Kurume, Japan \\ 2) Dental and Oral Medical Center, Kurume University School of Medicine, Kurume, Japan \\ 3) Advanced Imaging Research Center, Kurume University School of Medicine, Kurume, Japan \\ 4) Ube Industries, Ltd. Corporate Research and Development, Hagihara Research Group, Ube, Japan \\ (Accepted for publication, April 16, 2018)
}

\begin{abstract}
The regeneration of large bone defects is limited by reduced angiogenesis and cell migration from the remaining bone and tissue. Although scaffolds are required as autogenous bone grafts or artificial substrates for efficient bone tissue engineering, cellular implants can also facilitate bone regeneration, and bone marrow is frequently applied to treat such defects. Here, we attempted to reconstruct a three-dimensional hematopoietic environment on a newly developed porous polyimide membrane using a modified Dexter culture method and found that the membrane could maintain CD34-, CD45-, and TER-119-positive cells. We further applied membrane-enclosed, hematopoietic-lineage cells to a rat calvarial bone defect model to evaluate the effects of a multicellular environment on bone regeneration. Our results suggested that the cultured hematopoietic environment on the porous membrane facilitated new bone formation. The bone volume and bone mineral content values of the coculture group at 8 weeks post-surgery were significantly higher than those in samples where only bone marrow stromal cells were used. Thus, coculture with multiple cell types accelerated bone formation, and culturing diverse cells on a membrane may facilitate cell transplantation in bone tissue engineering.
\end{abstract}

Key words: Polyimide, Dexter culture, Bone marrow, Transplantation, Bone regeneration

\section{Introduction}

The reconstruction of large bone defects after trauma, tumor resection, inflammation, and periodontitis is a major clinical problem. Standard treatments for such defects include reconstruction and replacement using autogenous bone grafts or artificial bone, combined with titanium mesh. In recent years, stem cell therapy has been widely studied for developing basic and clinical applications in bone tissue engineering ${ }^{1-3}$. Although bone tissue engineering with stem cell therapy has been attempted, the size of bone regeneration is often limited by a lack of blood vessels; this prevents sufficient nutritional support to the whole bone graft $^{4}$. Consequently, a source of cells and angiogenesis around defects is considered essential for efficient bone regeneration ${ }^{5-7)}$, in addition to effective autogenous bone grafts and artificial substrates for use as a scaffold.

Coculture techniques are often applied in biology to study natural or synthetic interactions between cell populations ${ }^{8}$. In vitro stromamediated hematopoiesis has been studied using the Dexter culture system $^{9-13)}$. In this system, bone marrow mesenchymal stem cells (also known as bone marrow stromal cells [BMSCs] $)^{14,15}$ form an adherent layer, to which hematopoietic cells attach and support the maintenance of hematopoietic stem cells ${ }^{9-11,16}$. This contact between the stromal and hematopoietic cells is essential for hematopoiesis in vitro.

Some studies recently cocultured endothelial progenitor cells with BMSCs for bone tissue engineering ${ }^{17-19}$. They demonstrated that these cells could form a pre-vascular network during coculture, resulting in increased osteoblastic activity. CD34-positive cells, including endothelial progenitor and hematopoietic stem cells, are

Correspondence to: Dr. Keisuke Ohta, Division of Microscopic and Development Anatomy, Department of Anatomy, Kurume University School of Medicine, 67 Asahi-machi, Kurume City, Fukuoka 830-0011, Japan; Tel: +81-942-31-7541, Fax: +81-942-31-7555; E-mail: kohta@med.kurume-u. ac.jp capable of inducing neovascularization ${ }^{20-23)}$ and can differentiate into osteoblasts $^{24-27)}$. Matsumoto et al. ${ }^{24)}$ reported that CD34-positive cells have potent vasculogenic and osteogenic plasticity in the fractureinduced environment, enabling them to make major contributions to morphological and functional bone healing. Li et al. ${ }^{19)}$ reported that the use of a cocultured cellular sheet, comprised of BMSCs and CD34positive cells, for treating bone defects could promote bone regeneration more efficiently than a BMSC sheet alone. However, the procedure for sorting of CD34-positive cells is likely to increase the complexity of the method. The effects of coculturing BMSCs and other hematopoietic progenitor cells on bone regeneration are unclear, even though bone regeneration is known to involve hematopoiesis, neovascularization, and bone formation ${ }^{6}$.

In addition to collagen products, various polymer membranes are widely used in tissue engineering. Polymers are used in medical devices and implants ${ }^{28,29)}$. In particular, polyimides are high-performance polymers that are highly stable and biocompatible ${ }^{30)}$. Previously, we produced a novel polyimide porous membrane having different surface textures and opening diameters between the front and back sides, and evaluated its biocompatibility and effects on the bone formation rate in a rat calvarial defect model $^{311}$.

In this study, we attempted to generate a hematopoietic environment on the double-faced porous membrane of a polyimide membrane by the Dexter culture method and found that the membrane could maintain hematopoietic properties in vitro. We successfully applied the cellcultured membrane to a rat calvarial defect model to investigate the effects of the hematopoietic microenvironment on a polyimide porous membrane, to increase new bone formation and bone mineral density.

\section{Materials and Methods}

Animals and experimental design

All experiments were performed in accordance with the National 
J.Hard Tissue Biology Vol. 27(3): 185-194, 2018

Institutes of Health Guidelines for animal research. All of the animal procedures were approved by the Board for Animal Experiments at Kurume University (Admission Number: 2017- 123).

\section{In vitro study}

BMSCs from C57BL/6J mice ( $\mathrm{n}=6$; 9 weeks of age) and C57BL/6$\operatorname{Tg}$ (CAG-EGFP) mice $(\mathrm{n}=3 ; 10$ weeks of age) were isolated and cultured. Wild-type BMSCs were divided into BMSC and Dexter groups. Then, GFP-positive BMSCs were cultured on polyimide membranes for 7 days (Dexter culture). Each group was evaluated at 7 and 14 days by FIB/SEM and immunohistochemistry.

\section{In vivo study}

Nine-week-old F344/NJcl-rnu/rnu mice $(\mathrm{n}=30)$ were subjected to calvarial defect surgery, and 60 defects were assessed. Nine-weekold SD-Tg (CAG-EGFP) rats $(\mathrm{n}=3)$ were subjected to donor cell transplantation. Rats with calvarial defects were divided into the BMSC group (only BMSCs were transplanted with the polyimide membrane), the Dexter group (cells cultured by Dexter culture were transplanted with the polyimide membrane), and the control group (only the polyimide membrane was transplanted) $(n=10 /$ group $)$. In each group, four rats were subjected to micro-CT analysis and six rats were subjected to histological analysis. All rats were evaluated at 2, 4, and 8 weeks after calvarial defect surgery. Bone-regeneration changes over time (in vivo imaging) were observed by micro-CT with four rats from each group. At each time point, six rats from each group were sacrificed with sodium pentobarbital $(50 \mathrm{mg} / \mathrm{kg})$ and were transcardially perfused through the left ventricle with fixative solution, and tissues were observed after hematoxylin and eosin staining.

\section{Double-faced porous polyimide membrane preparation}

Membranes were prepared following the Loeb-Sourirajan $\operatorname{method}^{32,33)}$. First, polyamic acid solution was coated onto a stainlesssteel plate. The polyamic acid-coated plate was submerged into a coagulating bath and incubated for several minutes. During this process, porous polyamic acid developed on the plate as a white membrane.

This white membrane was recovered from the plate by transferring it from the aqueous, organic incubation bath into a fresh water bath for an additional incubation period. This precursor membrane was recovered, dried, and affixed to a pin tenter for drying. The pin tenter was placed into an electric furnace. The polyamic thin membrane was heated to $360^{\circ} \mathrm{C}$ to catalyze porous polyimide membrane formation.

To confirm production of the porous membrane, a small sample of the heat-treated membrane was placed on conductive double-stick tape with no additional conductive coating and observed using low-vacuum SEM (65 Pa). The double-faced porous polyimide membrane was used as a scaffold for cell culture. One side of the membrane had 40-60$\mu \mathrm{m}$ pores (void side), and the other side had $10-30-\mu \mathrm{m}$ pores (mesh side), with a wall-like structure connecting both surfaces (Fig. 1). The thickness of the membrane was approximately $25 \mu \mathrm{m}$. The membrane had internal macrovoids and high thermostability, and no alterations were observed after autoclave sterilization. Before use, the membranes were trimmed to $15 \times 15 \mathrm{~mm}$ and autoclaved $\left(121^{\circ} \mathrm{C}, 20 \mathrm{~min}\right)$.

\section{Cell isolation and culture on the polyimide porous membrane}

Bone marrow cell isolation was performed as described previously ${ }^{34)}$. At 9 weeks of age, bone marrow was obtained from C57BL/6J mice for in vitro study or from F344/NJcl-rnu/rnu rats for in vivo study. The femora were excised aseptically, cleaned of soft tissue, and washed four times (10 min each) in Dulbecco's modified Eagle's medium containing $10 \%$ fetal bovine serum and a mixed antibiotic-antimycotic solution (Nacalai Tesque, Kyoto, Japan). The bone ends were removed, and the marrow was flushed out using $5 \mathrm{ml}$ of culture medium expelled from a $10-\mathrm{ml}$ syringe through a 20 -gauge needle with $70-\mu \mathrm{m}$ cell strainers
(BD Falcon, Franklin Lakes, NJ, USA). For preculturing BMSCs, the bone marrow cells were seeded onto polyimide membranes (mesh side) in 6-well plastic culture flasks (BD Falcon) at $1 \times 10^{6}$ cells/well and cultured for 5 days at $37^{\circ} \mathrm{C}$ in a humidified atmosphere containing $5 \% \mathrm{CO}_{2}$. The medium was changed on day 6 . In the BMSC group, the preculture membrane was further cultured for 1 more week (for a total of 14 days from the beginning of culture). The medium was refreshed every 3 days after day 6 . In the Dexter group, newly prepared bone marrow cells were further added on day 8 as follows. Bone marrow cells for coculture were obtained from 10 -week-old C57BL/6-Tg (CAG-EGFP) mice for the in vitro study or 10-week-old SD-Tg (CAGEGFP) rats for the in vivo study, and $1 \times 106$ isolated bone marrow cells were added to the precultured polyimide membrane. The Dexter group membranes were further cultured for an additional 7 days (14 days total; Fig. 2).

\section{FIB/SEM tomography of cultured membranes}

Specimens were prepared for FIB/SEM tomography as described previously ${ }^{35-37)}$, with some modifications. On days 7 and 14 , the cell cultured membranes were fixed with $2 \%$ paraformaldehyde and incubated in $2.5 \%$ glutaraldehyde in $0.1 \mathrm{M}$ cacodylate ( $\mathrm{pH} 7.3$ ) buffer for $15 \mathrm{~min}$ at room temperature for fixation. The specimens were then washed three times with cacodylate buffer (10 min each). Next, the specimens were cut into small rectangles and subjected to post-fixation and en bloc staining. Briefly, after three washes in cacodylate buffer, the specimens were post-fixed for $1 \mathrm{~h}$ at $4^{\circ} \mathrm{C}$ in a solution containing $2 \%$ osmium tetraoxide and $1.5 \%$ potassium ferrocyanide in cacodylate buffer. The specimens were then washed three times with distilled water and immersed in $1 \%$ thiocarbohydrazide solution for $30 \mathrm{~min}$. After five washes with distilled water, the specimens were immersed in distilled water containing $2 \%$ osmium tetraoxide and washed three times with distilled water. The specimens were stained en bloc in a solution of $4 \%$ uranyl acetate dissolved in distilled water overnight for contrast enhancement, after which they were washed with distilled water. Next, the specimens were stained with Walton's lead aspartate solution for $1 \mathrm{~h}$, dehydrated in an ethanol series $(25 \%, 50 \%, 70 \%, 80 \%, 90 \%$, and twice in $100 \%$ ethanol; 5 min each), infiltrated with epoxy resin (Epon 812; TAAB, Aldermaston, UK), and polymerized for $72 \mathrm{~h}$ at $60^{\circ} \mathrm{C}$. The surfaces of the embedded specimens were exposed using a diamond knife on an Ultracut E microtome (Leica, Wetzlar, Germany). The resin blocks were then trimmed down and placed on a holder. For the 3D reconstruction, serial images of the block faces were acquired by repeated cycles of sample-surface milling and imaging using Slice \& View G2 operating software (FEI, Eindhoven, Netherlands). Milling was performed with a gallium ion beam at $30 \mathrm{kV}$ with a current of $15 \mathrm{nA}$. The milling pitch was set to $100 \mathrm{~nm} / \mathrm{step}$ and 600 cycles. The images were acquired at a landing energy of $2.5 \mathrm{keV}$. Additional acquisition parameters were as follows: beam current $=50.8 \mathrm{pA}$, dwell time $=6 \mu \mathrm{s} /$ pixel, image size $=2048 \times 1768$ pixels, and pixel size $=36$ $\mathrm{nm} / \mathrm{pixel}$. The resulting image stack and reconstruction were processed using Fiji software (http://fiji.sc/Fiji) and Amira 6.0.1 software (FEI Visualization Science Group, Burlington, MA, USA). Images were observed with optional $\mathrm{X}-\mathrm{Y}-\mathrm{Z}$ plane sectioning.

\section{Immunohistochemical staining of cultured polyimide membranes}

At each time point, specimens were fixed for 15 min with $4 \%$ paraformaldehyde at room temperature and washed three times with phosphate-buffered saline (PBS; 5 min each wash). For fluorescent immunostaining, Triton-X100 (0.2\% in PBS) was applied for $5 \mathrm{~min}$ at room temperature. The specimens were then washed with PBS three times for $5 \mathrm{~min}$. Subsequently, the specimens were incubated with PBS containing 5\% normal goat serum for $30 \mathrm{~min}$ at room temperature. For immunohistochemical staining, cultured polyimide membranes were immunostained with rat monoclonal antibodies against mouse CD34 
Shingo Hirashima et al:: An in Vitro Reconstructed 3D Hematopoietic Microenvironment for Bone Regeneration in Rats

void side
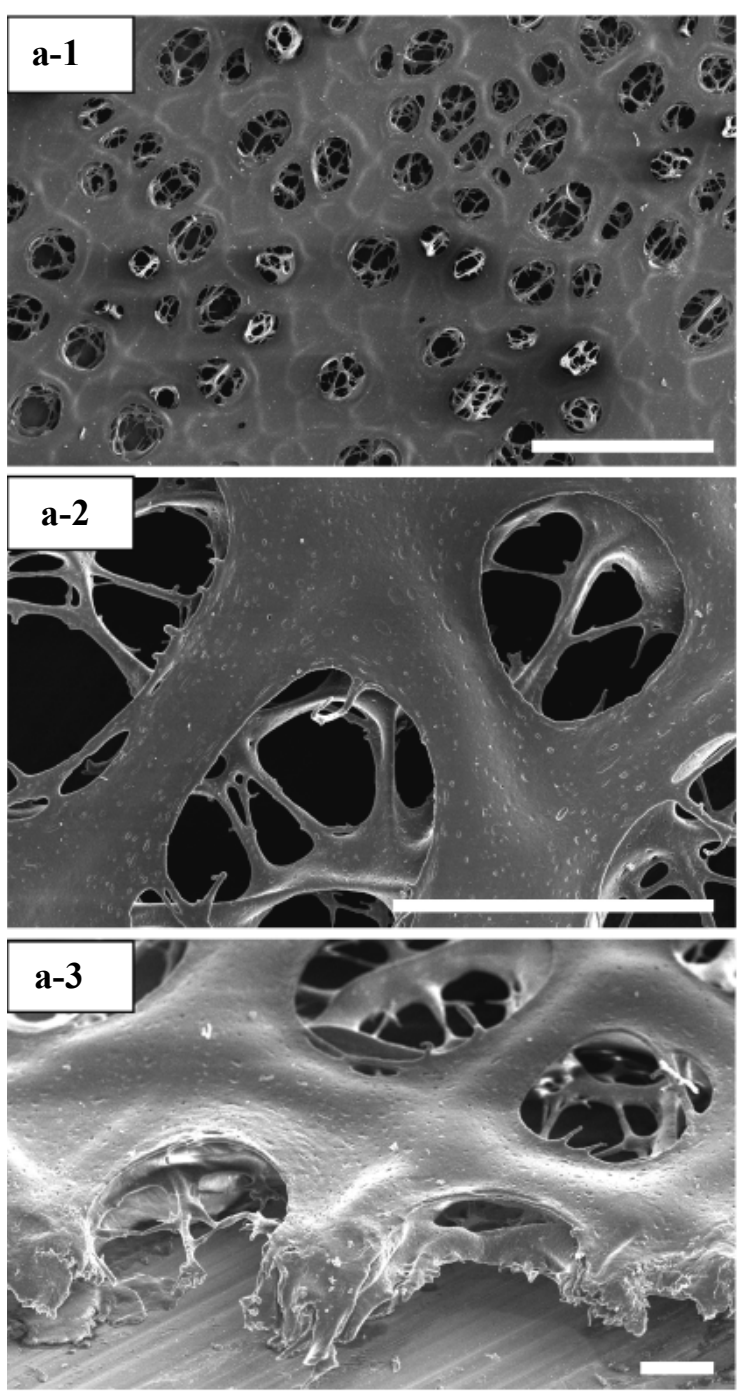

mesh side
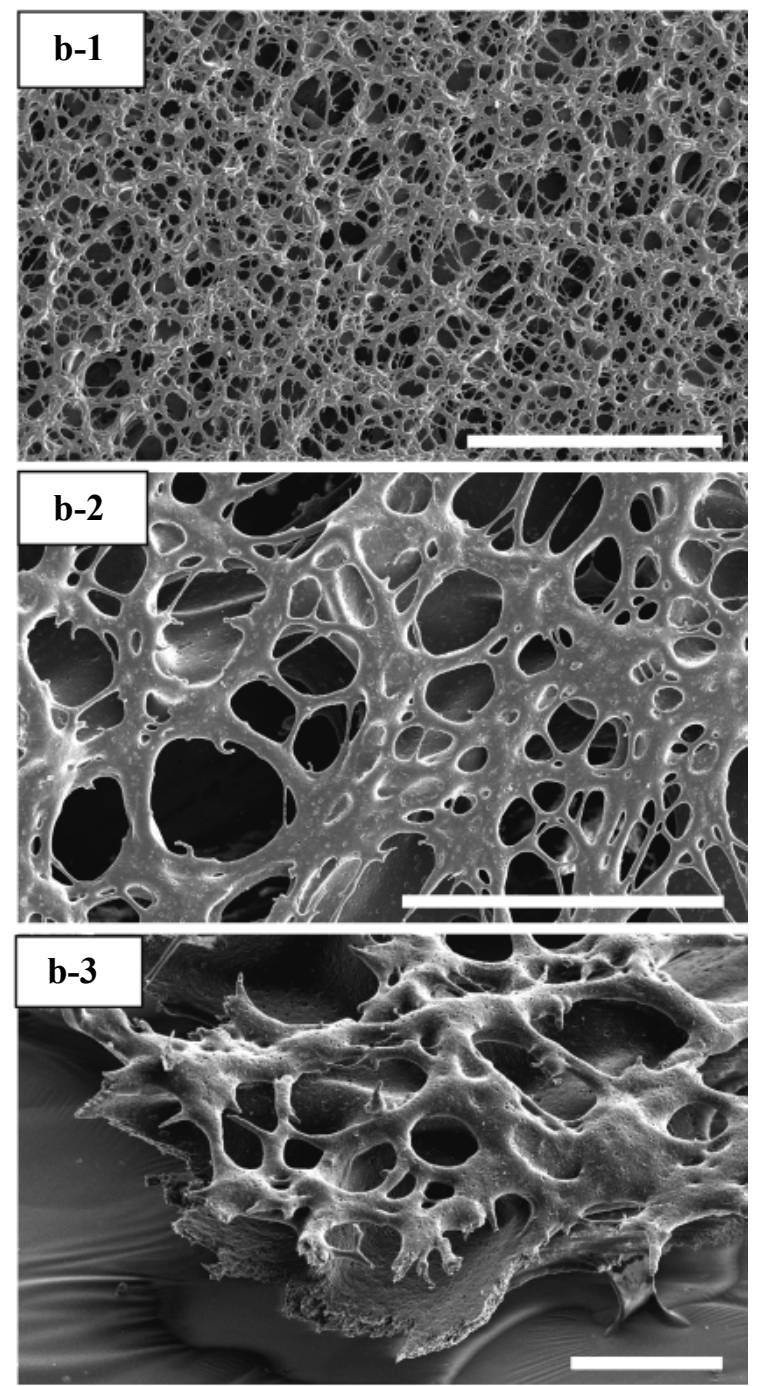

Figure 1. Double-faced porous polyimide membranes. Asymmetric porous polyimide membranes had $40-60-\mu \mathrm{m}$ pores on the void side (a-1-3) and $10-30-\mu \mathrm{m}$ pores on the mesh side (b-1-3). A wall-like structure connected the two surfaces. Scale bars: $200 \mu \mathrm{m}$ for panel (a-1.b-1), $50 \mu \mathrm{m}$ for panel (a-2.b-2), and $20 \mu \mathrm{m}$ for panel (a-3.b-3).

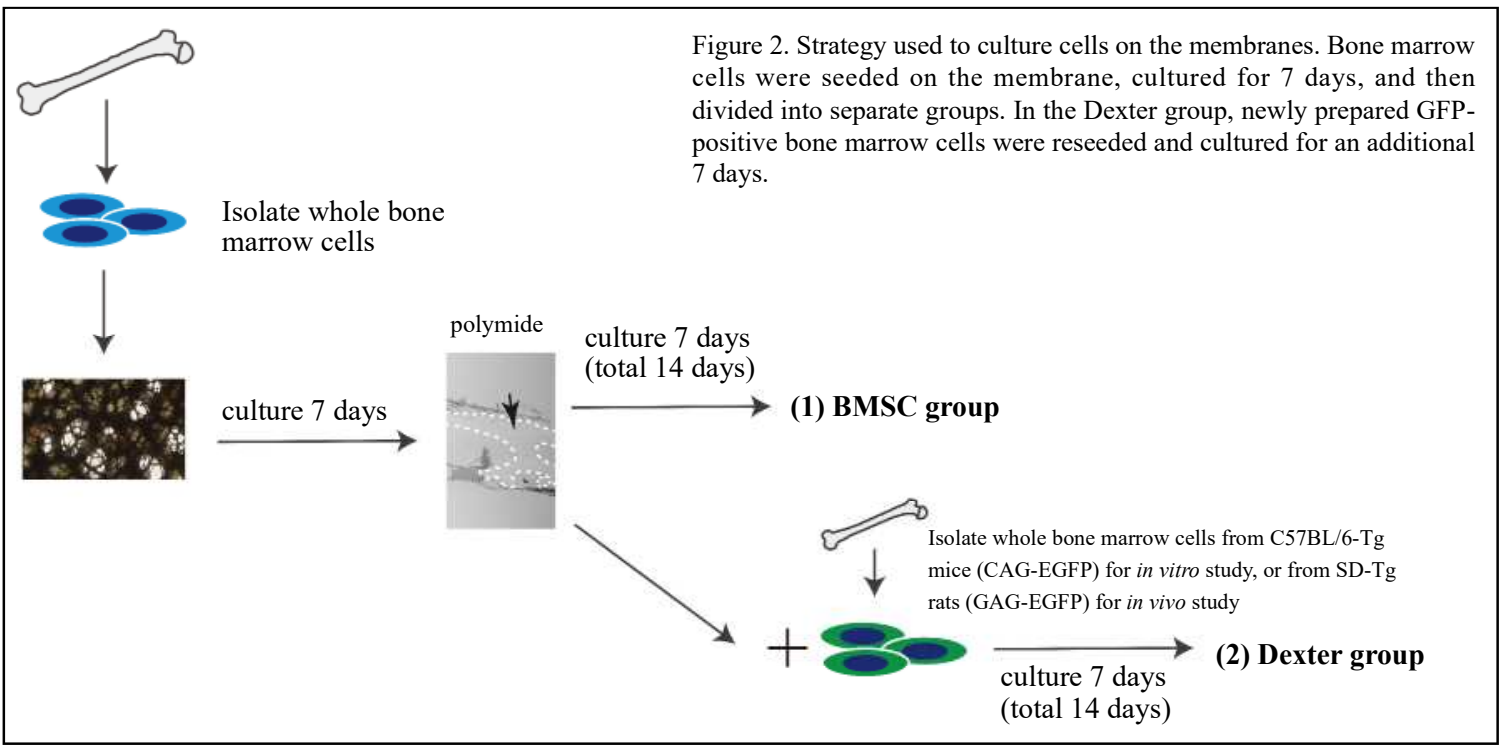



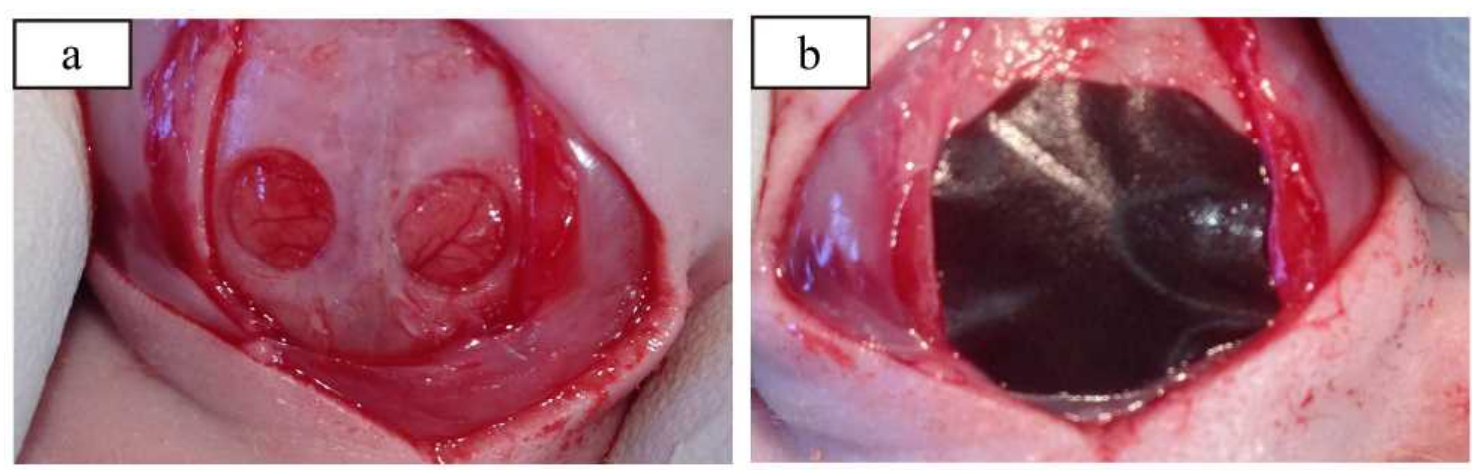

Figure 3. Surgical procedures used for membrane implantation on the bone defect. Two circular bone defects (full thickness, 4 mm in diameter) were made in the calvarial bone using a trephine bur (a). The defect was covered with a polyimide membrane (b).
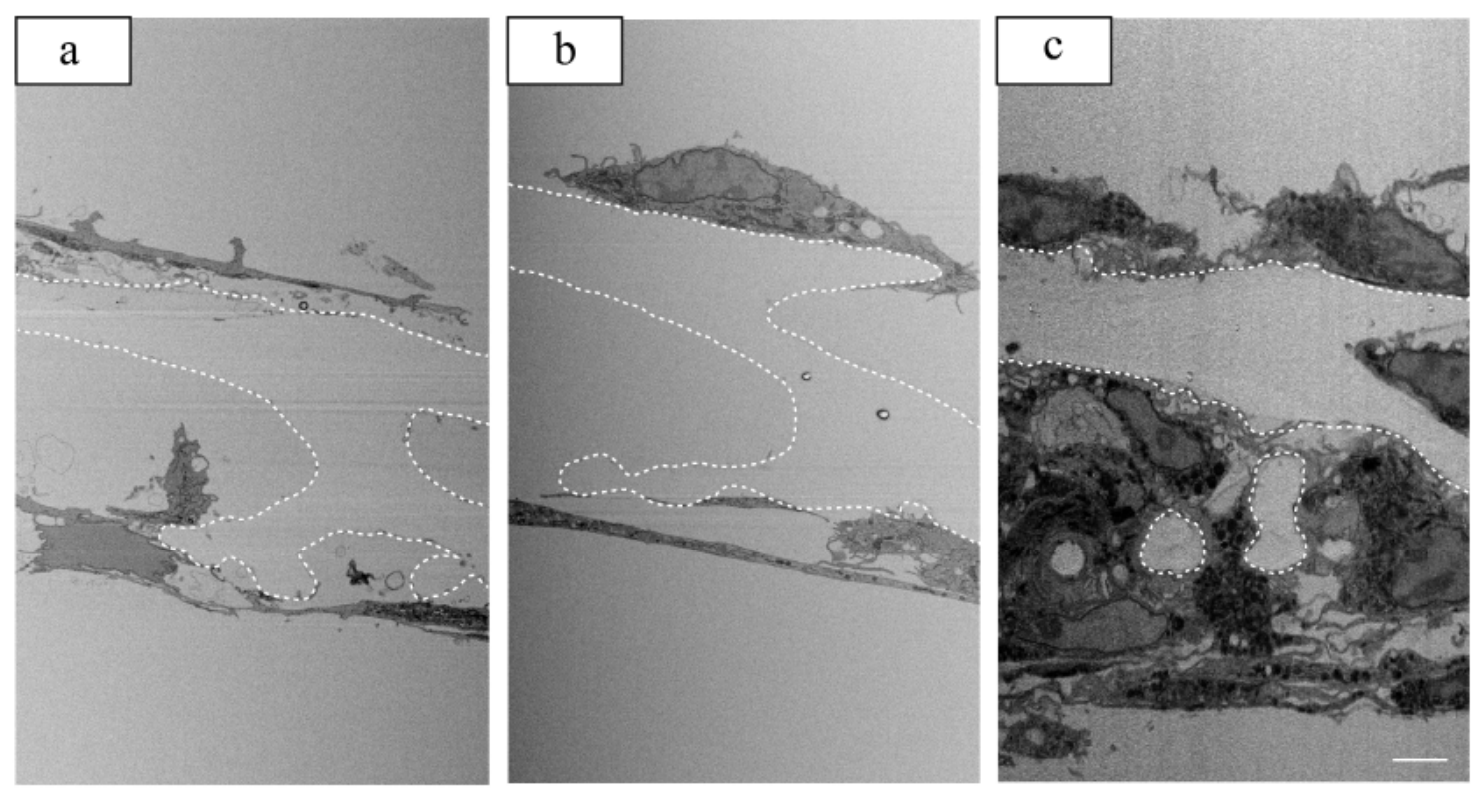

Figure 4. FIB/SEM images of membrane cross-sections (the polyimide substrate is surrounded by dotted lines). Day-7 (a) and BMSC-group (b) membranes, which were seeded with crude bone marrow cells and cultured for 7 or 14 days, respectively, showed flat cells on the polyimide membrane. The Dexter culture group (c), in which the membrane was reseeded with newly prepared bone marrow cells on a membrane from the same batch as the control group (day 7) and further cultured for 7 days, showed densely packed cells with abundant cellular organelles and cytoplasm that adhered to flat cells. Scale bars: $5 \mu \mathrm{m}$.

(1: 800 dilution; RAM34, eBioscience, San Diego, CA, USA), mouse CD45 (1: 1500 dilution; YW62.31, AbD Serotec, Oxford, UK), and mouse Ly76 (1: 100 dilution; TER-119, Abcam, Cambridge, UK). The specimens were incubated with a primary antibody for $1 \mathrm{~h}$ at room temperature, followed by $1 \mathrm{~h}$ at room temperature with Alexa Fluor 647 goat anti-rabbit secondary antibody (Invitrogen, Carlsbad, CA, USA). The specimens were counterstained with 4',6-diamidino-2-phenylindole (DAPI; 1: 2000; Wako, Kumamoto, Japan) to define the nuclei, and PermaFluor (Thermo Fisher Scientific, Waltham, MA, USA)-mounted sections were observed with a confocal laser scanning microscope (FluoView, FV1000D; Olympus, Tokyo, Japan).

\section{Preparation of polyimide membranes for transplantation}

On the day before transplantation, polyimide membranes for the BMSC and Dexter groups were washed three times with PBS and changed to serum-free medium.

\section{Surgical procedures}

F344/NJcl-rnu/rnu rats were anesthetized with isoflurane under a high flow rate of oxygen. The surgical site was shaved and treated with a $70 \%$ ethanol solution, and a subcutaneous injection of $0.25 \mathrm{ml}$ of $2 \%$ lidocaine (local anesthetic) was administered at the sagittal (middle) skull section. A sagittal incision was made over the scalp from the nasal bone to the middle sagittal crest, and the periosteum was opened to expose the surface of the calvarial bone. Two circular bone defects (full thickness, 4-mm diameter) were made in the calvarial bone using a trephine bur, and irrigated with saline to remove the bone debris. The animals were randomly assigned into the following three groups based on the type of covering membrane: BMSC group, Dexter group, and control group. The skin was sutured with 4-0 nylon (Fig. 3).

\section{Micro-CT analysis of the calvarial-defect model}

At 2, 4, and 8 weeks post-operation, micro-CT was used to quantify the volume of bone formation within each defect. The animals $(n=4$, eight defects/group) were anesthetized with isoflurane. Living rats were positioned such that the coronal aspect of the calvarial bone was in a horizontal position, and the calvaria were scanned using micro-CT ( $\mathrm{R}_{-}$ mCT2, Rigaku, Tokyo, Japan) at $90 \mathrm{kV}$ and $180 \mu \mathrm{A}$. The images were analyzed using TRI/3D-Bone (RATOC, Tokyo, Japan). A cylindrical volume of interest (4-mm diameter, 1.18-mm height) was selected to calculate the BMC, BV, and BMD values of the regenerated bones in the three groups. 
Shingo Hirashima et al:: An in Vitro Reconstructed 3D Hematopoietic Microenvironment for Bone Regeneration in Rats

DAPI

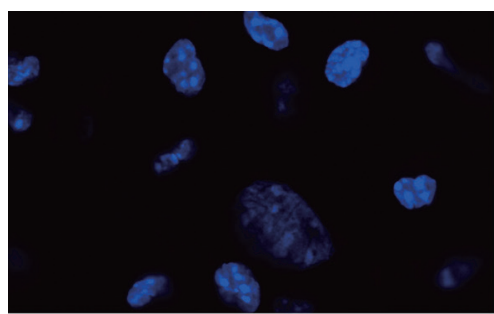

GFP

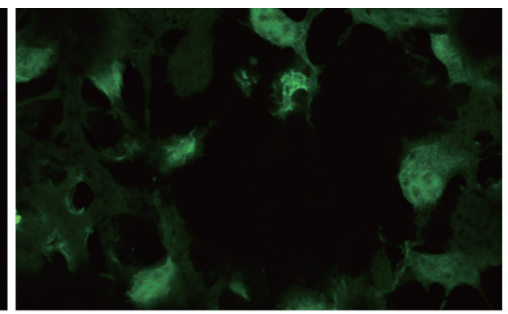

Merge

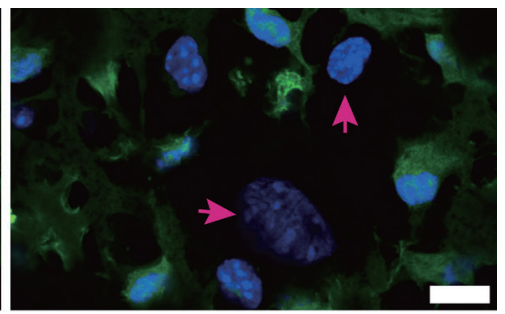

Figure 5. Cell culture on asymmetric porous polyimide membranes. GFP-positive and -negative cells (magenta arrows) were observed in polyimide porous membranes in the Dexter culture group. Scale bars: $5 \mu \mathrm{m}$.

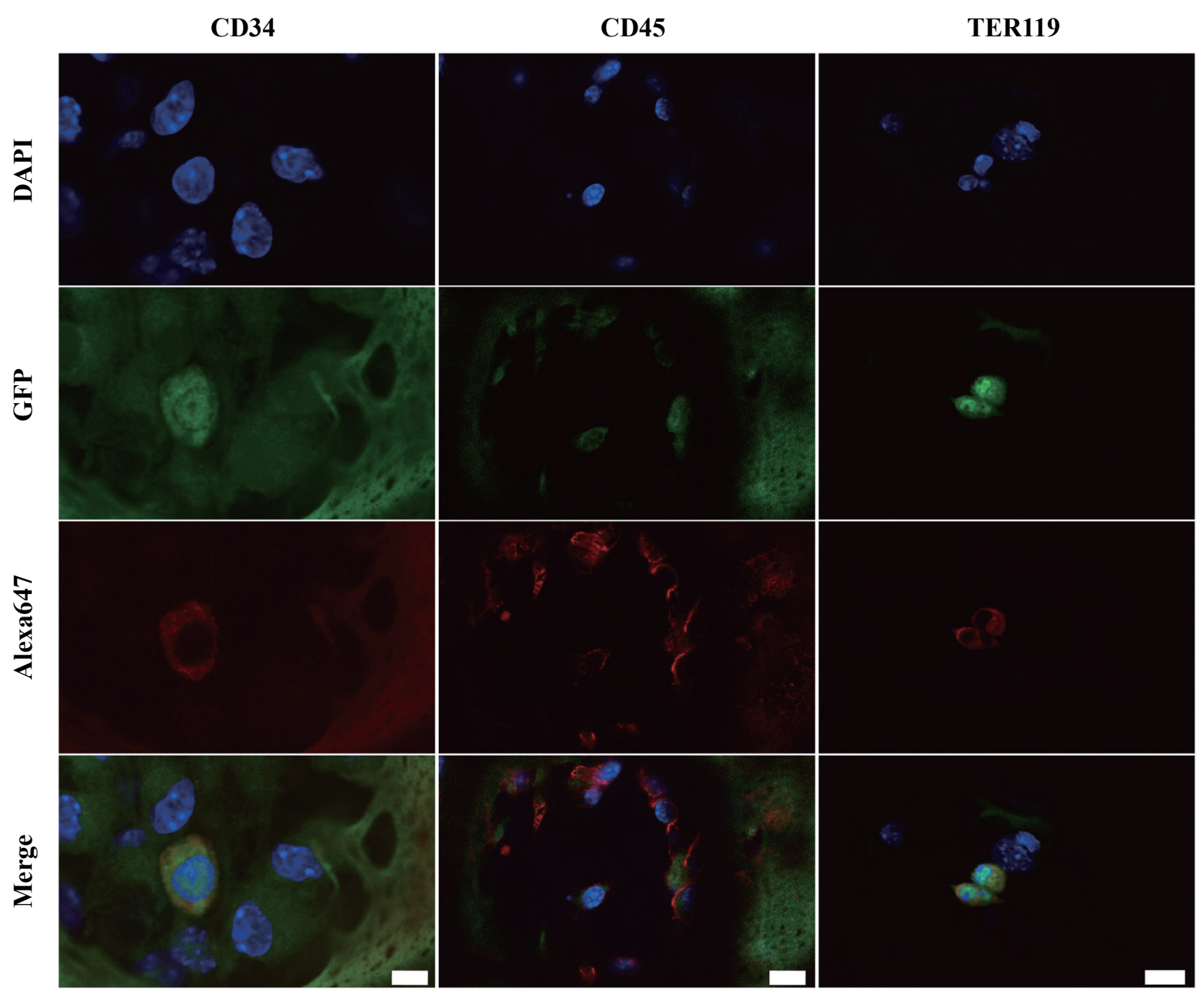

Figure 6. Immunocytochemistry of bone marrow cell cultures on polyimide membranes. In the Dexter culture group, GFP-positive cells were also positive for CD34, CD45, and TER-119. Scale bars: $10 \mu \mathrm{m}$.

\section{Histological analysis of the rat calvarial-defect model}

At 2,4 , and 8 weeks post-operation, the animals $(\mathrm{n}=2$, four defects/group) were deeply anesthetized with diethyl ether and sodium pentobarbital $(50 \mathrm{mg} / \mathrm{kg})$, transcardially perfused through the left ventricle with heparin-containing $(10 \mathrm{U} / \mathrm{ml})$ saline, and fixed with $4 \%$ paraformaldehyde in PBS. After perfusion, the parietal bones containing the skin were removed from the skull using a saw. The specimens were then immersed in the same fixative for $2 \mathrm{~h}$ at $4^{\circ} \mathrm{C}$, rinsed in buffer, and decalcified in Kalkitox solution (Wako Pure Chemical Industries, Ltd., Osaka, Japan) for 5 days. Subsequently, the specimens were trimmed, washed three times for $5 \mathrm{~min}$ each in PBS, immersed in PBS containing $30 \%$ sucrose for 3 days at $4^{\circ} \mathrm{C}$, and frozen in Tissue-Tek OCT compound (Sakura Finetek, Torrance, CA, USA). Frozen blocks were cut into 6- $\mu \mathrm{m}$-thick sections using a cryostat (CryoMicrotome CM1950; Leica, Wetzlar, Germany). The sections were then observed after hematoxylin and eosin staining.

\section{Statistical analysis}

Statistical analysis was performed using JMP software, version 13 (SAS Institute Inc., Cary, NC, USA). Kruskal-Wallis tests with Wilcoxon's post-hoc analyses were used to evaluate three areas by comparing the BMD, BMC, and BV. Values are shown as the mean \pm standard deviation (SD). $\mathrm{p}<0.05$ was considered statistically significant. 


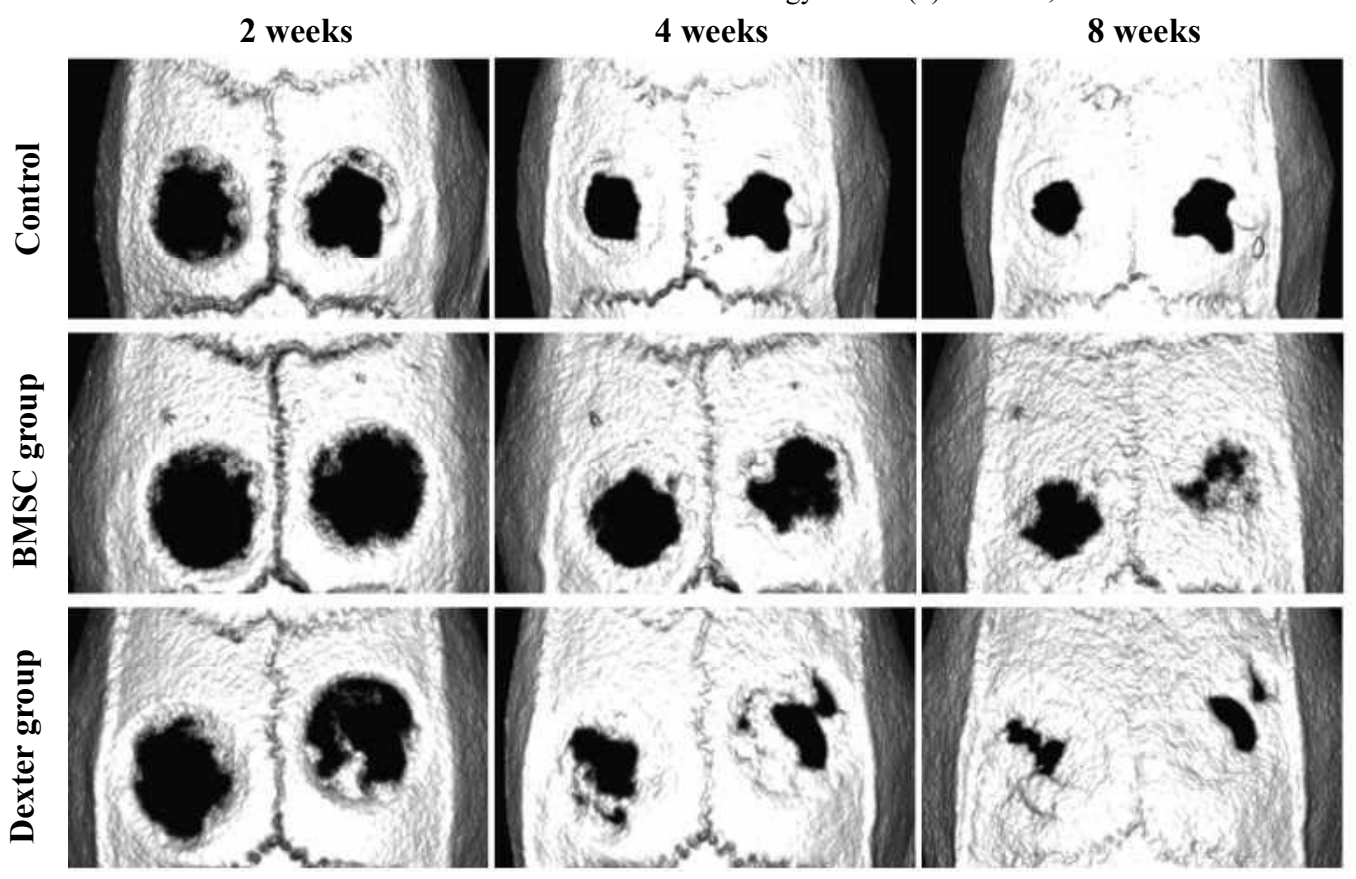

Figure 7. 3D-CT imaging. Bone regeneration was observed by $3 \mathrm{D}-\mathrm{CT}$. New bone formation in each group was observed at the defect border. At 4 and 8 weeks, the Dexter group showed a larger amount of new bone formation than the other groups. No group showed fully regenerated bone within 8 weeks.

a
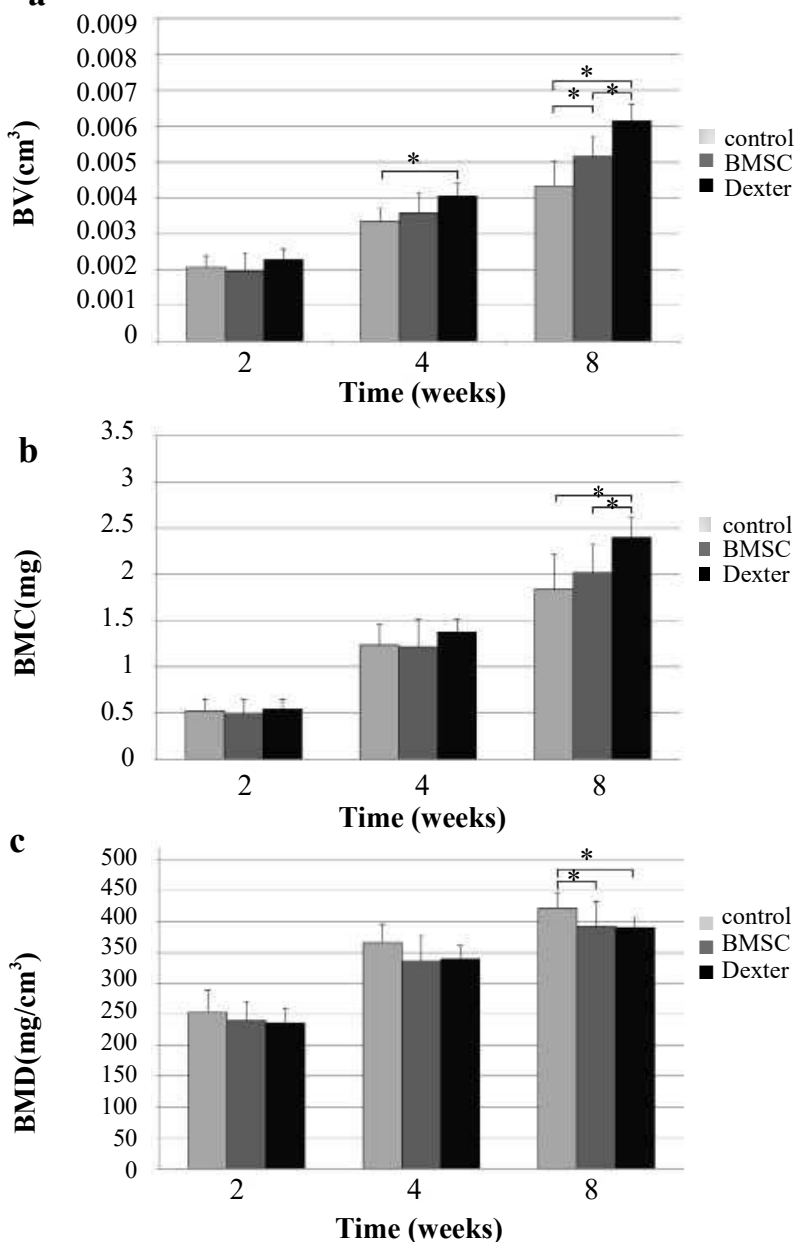

Figure 8. Micro-CT analysis. At 2, 4, and 8 weeks post-operation, micro$\mathrm{CT}$ was used to determine the bone mineral density (BMD (a)), bone mineral content (BMC (b)), and bone volume (BV (c)) of the regenerated bone in the three groups. Error bars represent the standard deviation. Asterisks denote statistically significant differences $(\mathrm{p}<0.05)$.

\section{Results}

Focused ion beam/scanning electron microscopy (FIB/SEM) observations

Cross-sections of day-7 precultured membranes showed many flat cells on the polyimide membrane, but few cells were observed in the porous space of the membrane (Fig. 4a). The cells on the membrane had a thin cytoplasm, and the organelles were not well developed. The features of BMSCs cultured on the membrane (Fig. 4a) were similar to those on the precultured membrane (Fig. 4b). Many flat and thin cells on the membrane surface were observed in the cross-sections; however, few cells were observed in the porous space, even on day 14 (Fig. $4 b$ ). In contrast, in the Dexter group, in which newly prepared bone marrow cells were added to the precultured membrane on day 7 and further cultured for 1 more week, numerous cells were observed in the porous space of the membrane (Fig. 4c). Each cell was rich in terms of its cytoplasm and cellular organelles, and had many cellular processes; these cells adhered to flat cells on the membrane surface (Fig. 4c).

\section{Immunocytochemistry of bone marrow cell cultures on polyimide membranes}

In the Dexter group, both green fluorescent protein (GFP)-positive and -negative cells were observed (Fig. 5). GFP-positive cells were derived from the reseeded bone marrow cells. Immunocytochemical analysis of the membrane showed immunoreactivity for CD34, CD45, and TER-119 on GFP-positive cells (Fig. 6), whereas GFP-negative cells were negative for CD34, CD45, and TER-119. With precultured membranes on day 7 and the BMSC group, we did not observe immunoreactivity against CD34, CD45, or TER-119 in GFP-positive cells.

\section{Micro-X ray computed tomography (micro-CT) analysis of the calvarial defect model}

Pieces of polyimide porous membrane cultured with bone marrow cells were transplanted to calvarial bone defects, and the bone volume (BV), bone-mineral content (BMC), and bone-mineral density (BMD) in each defect area were assessed at 2, 4, and 8 weeks after surgery, using micro-CT (Fig. 7). The BVs of the control group, BMSC group, and Dexter group are shown in Fig. 8a. The BV of the BMSC group 
Shingo Hirashima et al:: An in Vitro Reconstructed 3D Hematopoietic Microenvironment for Bone Regeneration in Rats
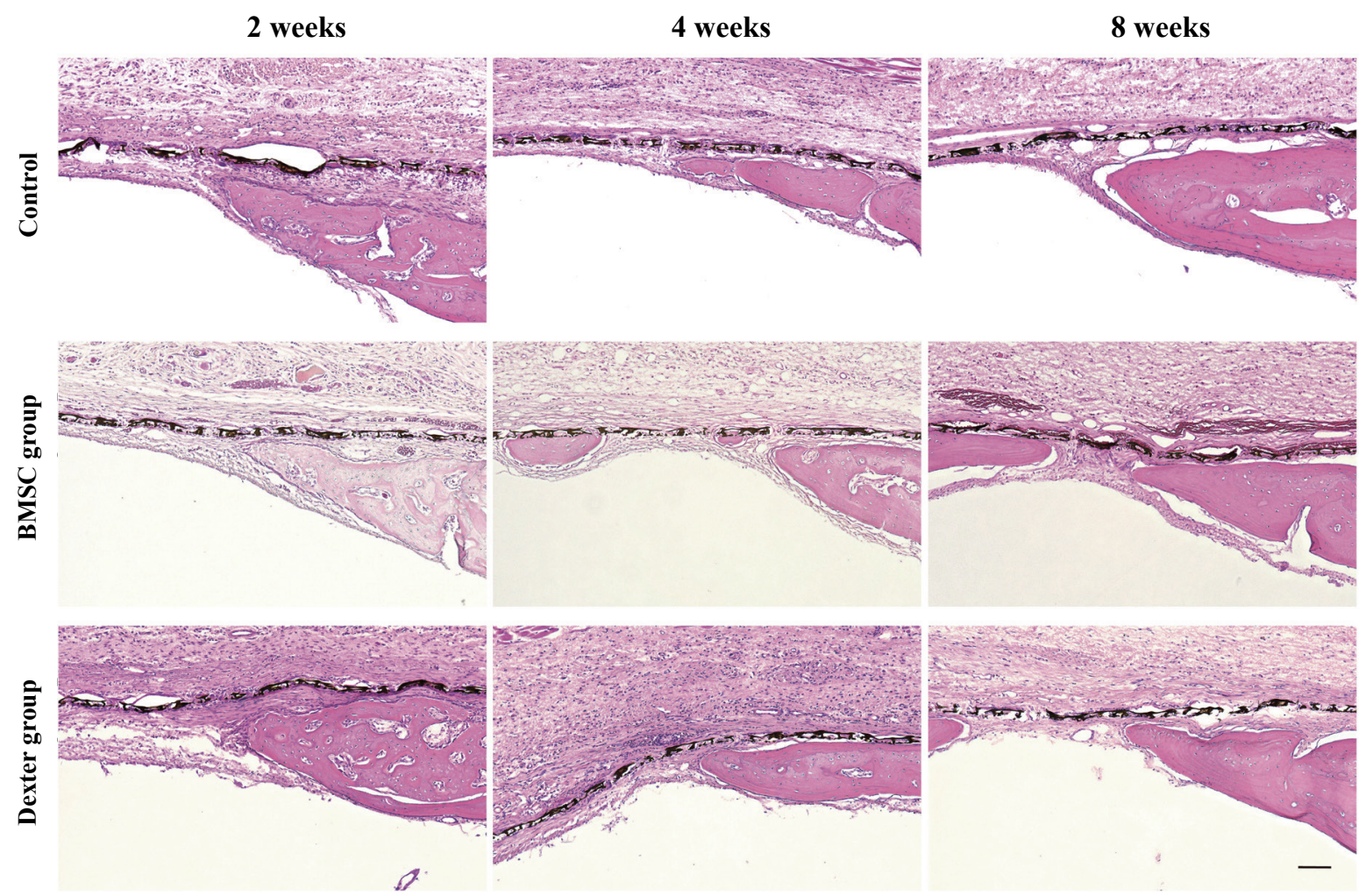

Figure 9. Histological evaluation of the border area of the newly regenerated bone. In the BMSC and Dexter groups, new bone was regenerated both at the margin of the existing bone and around the polyimide membrane. In the control group, new bone was regenerated only at the margin of the existing bone. Scale bar: $100 \mu \mathrm{m}$.

BMSC group at 8 weeks after surgery

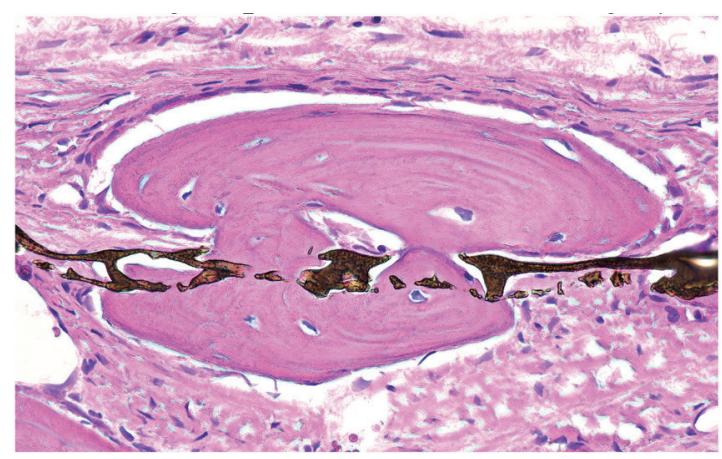

Dexter group at 8 weeks after surgery

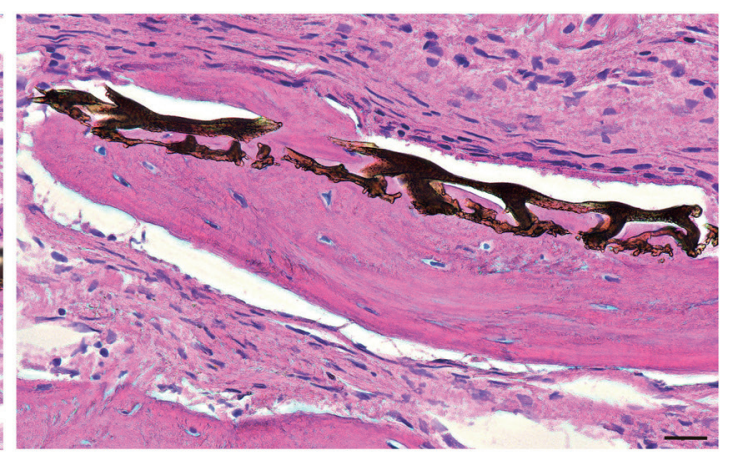

Figure 10. High-magnification images of regenerated bone around the polyimide membrane. New bone formation was observed inside the porous structure. Scale bar: $20 \mu \mathrm{m}$.

was significantly higher than that of the control group, but only at 8 weeks after surgery. Additionally, the BV of the Dexter group was significantly higher than that of the control group at 4 and 8 weeks after surgery and was significantly higher than that of the other groups at 4 weeks after surgery (Fig. 8a). The BMC of the Dexter group at 8 weeks after surgery was significantly higher than that of the other groups (Fig. $8 b)$. Finally, the BMD of the control group at 8 weeks after surgery was significantly higher than that of the other groups (Fig. 8c).

\section{Histological analysis of the rat calvarial defect model}

The number of cells surrounding the membrane gradually decreased over time in all groups (Fig. 9). Collagen bundles were observed in the defect area, and some of them passed through the porous membrane and appeared to mature over time. In the BMSC and Dexter groups, new bone formed, not only at the margin of the existing bone but also inside the porous space of the polyimide membrane (Fig. 10). In the control group, new bone formed only around the originally existing bone.

\section{Discussion}

Because polyimide has high biocompatibility and has been utilized for cell carriers and implants ${ }^{30,31,38-41)}$, we used the porous polyimide membrane as a cell source for bone regeneration around the defect. In this study, we applied a novel porous polyimide membrane for cell culture and succeeded in reconstructing the three-dimensional (3D) hematopoietic microenvironment by modifying the Dexter culture method. The defect created in the rat calvaria was a critical size defect and therefore could not heal spontaneously. We found that the hematopoietic environment was sustained using the porous membrane and that new bone formation was facilitated when we applied the membrane in a rat calvarial defect model. 
Although culturing simple crude bone marrow cells on the membrane did not suffice for maintaining hematopoietic cells, the Dexter culture method, in which crude bone marrow cells were reseeded on the precultured basement of bone marrow cells, enabled us to reconstruct the hematopoietic microenvironment. To evaluate the hematopoietic cell lineage on the membrane, we used antibodies against CD34 (a transmembrane phosphoglycoprotein, first identified on hematopoietic stem and progenitor cells), CD45 (a leukocyte common antigen expressed on most leukocytes, including hematopoietic progenitor cells), and TER-119 (a lineage marker for erythroid cells from early pro-erythroblasts to mature erythrocyte stages). In the single-culture groups (precultured-membrane and BMSC groups), no immunopositive cells were found on the membrane. Additionally, most cells on the membrane were spread wide and had a thin cytoplasm. These characteristics are consistent with the features of BMSCs. BMSCs are adhesive cells in the bone marrow and are easily isolated from crude bone marrow cells based on this feature; our results suggest that the BMSCs also adhered to the polyimide substrate. As shown by our experiments with the Dexter culture method, the hematopoietic cells may contact cultured BMSCs and help maintain their cellular characteristics. Our 3D Dexter culture method showed that the secondarily applied bone marrow cells were maintained on the original BMSCs and were CD34-, CD45-, and TER-119-positive hematopoietic cells. Additionally, these membranes could produce erythrocytes after the addition of cytokines (data not shown). These hematopoietic cells may exist within the porous space in the membrane, surrounding and interacting with the BMSCs. Therefore, we reconstructed a 3D hematopoietic microenvironment in the thin artificial membrane. Furthermore, the membrane may also maintain other types of stem cells in addition to the hematopoietic cells. A limitation of this study is that we only used one type of polyimide membrane, and further studies are needed to evaluate polyimide membranes of varying thicknesses and to determine the optimum thickness for achieving new bone formation. Our 3D culture demonstrated the applicability of the membrane as a cell carrier and may facilitate cell generation after transplantation to injured tissues.

In living organs, several different types of cells are related to bone healing ${ }^{6,42-44)}$. Therefore, the manipulation of cellular functions by coculture has been attempted to mimic the in vivo situation. Piracco et al. ${ }^{43)}$ suggested that endothelial cells are an indispensable component in bone tissue engineering, in addition to osteoblasts and osteoprogenitor cells. Mifune et al. ${ }^{26)}$ reported that transplanted CD34positive cells around the bone fracture facilitated vasculogenesis, increased the number of osteoblasts, and improved bone repair. The interactions between multiple types of cells dramatically increase the complexity of the reaction pathways, and such complexity is expected to help alleviate injury; however, preparing multi-cell cocultures is complicated, and assessing the associated molecular mechanisms is also quite difficult. Some reports have indicated that such complexity leads to unpredictable results ${ }^{8,45)}$. Notably, our 3D culture model enabled many cells to be maintained in a membrane using a relatively simple procedure. Additionally, improved $\mathrm{BV}$ and $\mathrm{BMC}$ results were obtained using Dexter-cultured membranes compared with simple BMSCcultured membranes. Therefore, our results suggested that bone tissue engineering using diverse cell species may be more effective. Li et al. ${ }^{19)}$ hypothesized that various cytokines and growth factors secreted from cocultured cells may exert greater paracrine effects than those from BMSCs, and we also expect that the hematopoietic microenvironment on the membrane would enable the secretion of various cytokines and facilitate intrinsic angio-osteogenesis. Further studies are needed to elucidate the composition and function of the cells that can be cultured on our polyimide porous membrane and to better understand the detailed processes supporting tissue formation with cultured cells using our culture system.
Micro-CT analysis showed that the BV and BMC values of the Dexter group were significantly higher than those of the other groups at 8 weeks after surgery. However, the BMD values were slightly lower than those of the control group. We employed micro-CT analysis in this study to assess the quality of regenerated bone. However, other assessments are needed to determine the clinical applications of the membrane, such as mechanical tests (e.g., functional assessment of a rotator cuff-repair mode ${ }^{1 / 46,47}$. For example, high-quality bone having appropriate strength is necessary for the success of treatment. Therefore, mechanical tests should be performed in a rat calvarial defect model in a future study.

The low BMD values observed in the BMSC and Dexter transplant groups may be explained by the lack of a scaffold. We have also confirmed that the polyimide porous membrane functions as a barrier membrane for guided bone regeneration ${ }^{48}$ and that the membrane itself facilitates bone regeneration; however, we expect that the combination with matrix components would improve the results. Therefore, further improvement of the BMD may be achieved by combining hydroxyapatite and other scaffolds with the currently described method. In summary, our findings revealed that coculturing BMSCs and hematopoietic progenitor cells via the Dexter culture method established a hematopoietic environment. Because the use of a cell carrier is essential for sustaining cell constructs in bone defects, our method increased the BV and BMC compared with transplanting BMSCs alone in a rat calvarial-defect model. Our findings suggest that coculturing of various cells related to bone formation is effective for improving bone regeneration and neovascularization in bone tissue engineering.

\section{Acknowledgement}

The authors thank Ms. Satoko Okayama and Mr. Akinobu Togo for their technical assistance. This research supported by research funding from Ube Industries, Ltd.

Conflict of Interest

Keisuke Ohta received research funding from Ube Industries, Ltd.

\section{References}

1. Aubin JE. Bone stem cells. J Cell Biochem Suppl 30-31: 73-82, 1998

2. Deans RJ and Moseley AB. Mesenchymal stem cells: biology and potential clinical uses. Exp Hematol 28: 875-884, 2000

3. Alghazali KM, Nima ZA, Hamzah RN, Dhar MS, Anderson DE and Biris AS. Bone-tissue engineering: complex tunable structural and biological responses to injury, drug delivery, and cell-based therapies. Drug Metab Rev 47: 431-454, 2015

4. Mikos AG, Herring SW, Ochareon P, Elisseeff J, Lu HH, Kandel R, Schoen FJ, Toner M, Mooney D, Atala A, Van Dyke ME, Kaplan $\mathrm{D}$ and Vunjak-Novakovic G. Engineering complex tissues. Tissue Eng 12: 3307-3339, 2006

5. Shieh SJ and Vacanti JP. State-of-the-art tissue engineering: from tissue engineering to organ building. Surgery 137: 1-7, 2005

6. Filipowska J, Tomaszewski KA, Niedzwiedzki L, Walocha JA and Niedzwiedzki T. The role of vasculature in bone development, regeneration and proper systemic functioning. Angiogenesis doi: 10.1007/s10456-017-9541-1, 2017

7. Borgiani E, Duda GN and Checa S. Multiscale modeling of bone healing: toward a systems biology approach. Front Physiol 8: 287, 2017

8. Goers L, Freemont P and Polizzi KM. Co-culture systems and technologies: taking synthetic biology to the next level. J R Soc Interface 11: 20140065, 2014

9. Dexter TM, Allen TD, Lajtha LG, Schofield R and Lord BI. Stimulation of differentiation and proliferation of haemopoietic cells in vitro. J Cell Physiol 82: 461-473, 1973 
Shingo Hirashima et al:: An in Vitro Reconstructed 3D Hematopoietic Microenvironment for Bone Regeneration in Rats

10. Dexter TM and Lajtha LG. Proliferation of haemopoietic stem cells in vitro. Br J Haematol 28: 525-530, 1974

11. Dexter TM, Allen TD and Lajtha LG. Conditions controlling the proliferation of haemopoietic stem cells in vitro. J Cell Physiol 91: 335-344, 1977

12. Wang TY, Brennan JK and Wu JH. Multilineal hematopoiesis in a three-dimensional murine long-term bone marrow culture. Exp Hematol 23: 26-32, 1995

13. Takagi M. Cell processing engineering for ex-vivo expansion of hematopoietic cells. J Biosci Bioeng 99: 189-196, 2005

14. Prockop DJ. Marrow stromal cells as stem cells for nonhematopoietic tissues. Science 276: 71-74, 1997

15. Lindner U, Kramer J, Rohwedel J and Schlenke P. Mesenchymal stem or stromal cells: toward a better understanding of their biology? Transfus Med Hemother 37: 75-83, 2010

16. Dexter TM. Stromal cell associated haemopoiesis. J Cell Physiol Suppl 1: 87-94, 1982

17. Yu H, Vandevord PJ, Gong W, Wu B, Song Z, Matthew HW, Wooley PH and Yang SY. Promotion of osteogenesis in tissueengineered bone by pre-seeding endothelial progenitor cellsderived endothelial cells. J Orthop Res 26: 1147-1152, 2008

18. Koob S, Torio-Padron N, Stark GB, Hannig C, Stankovic Z and Finkenzeller $\mathrm{G}$. Bone formation and neovascularization mediated by mesenchymal stem cells and endothelial cells in critical-sized calvarial defects. Tissue Eng Part A 17: 311-321, 2011

19. Li G, Wang X, Cao J, Ju Z, Ma D, Liu Y and Zhang J. Coculture of peripheral blood CD34+ cell and mesenchymal stem cell sheets increase the formation of bone in calvarial critical-size defects in rabbits. Br J Oral Maxillofac Surg 52: 134-139, 2014

20. Asahara T, Murohara T, Sullivan A, Silver M, van der Zee R, Li T, Witzenbichler B, Schatteman G and Isner JM. Isolation of putative progenitor endothelial cells for angiogenesis. Science 275: 964967, 1997

21. Asahara T, Masuda H, Takahashi T, Kalka C, Pastore C, Silver M, Kearne M, Magner M and Isner JM. Bone marrow origin of endothelial progenitor cells responsible for postnatal vasculogenesis in physiological and pathological neovascularization. Circ Res 85: 221-228, 1999

22. Mackie AR and Losordo DW. CD34-positive stem cells: in the treatment of heart and vascular disease in human beings. Tex Heart Inst J 38: 474-485, 2011

23. Sidney LE, Branch MJ, Dunphy SE, Dua HS and Hopkinson A. Concise review: evidence for $\mathrm{CD} 34$ as a common marker for diverse progenitors. Stem Cells 32: 1380-1389, 2014

24. Matsumoto T, Kawamoto A, Kuroda R, Ishikawa M, Mifune Y, Iwasaki H, Miwa M, Horii M, Hayashi S, Oyamada A, Nishimura H, Murasawa S, Doita M, Kurosaka M and Asahara T. Therapeutic potential of vasculogenesis and osteogenesis promoted by peripheral blood CD34-positive cells for functional bone healing. Am J Pathol 169: 1440-1457, 2006

25. Matsumoto T, Kuroda R, Mifune Y, Kawamoto A, Shoji T, Miwa M, Asahara T and Kurosaka M. Circulating endothelial/skeletal progenitor cells for bone regeneration and healing. Bone 43: 434439, 2008

26. Mifune Y, Matsumoto T, Kawamoto A, Kuroda R, Shoji T, Iwasaki H, Kwon SM, Miwa M, Kurosaka M and Asahara T. Local delivery of granulocyte colony stimulating factor-mobilized CD34-positive progenitor cells using bioscaffold for modality of unhealing bone fracture. Stem Cells 26: 1395-1405, 2008

27. Chen JL, Hunt P, McElvain M, Black T, Kaufman S and Choi ES. Osteoblast precursor cells are found in CD34+ cells from human bone marrow. Stem Cells 15: 368-377, 1997

28. Nair LS and Laurencin CT. Polymers as biomaterials for tissue engineering and controlled drug delivery. Adv Biochem Eng
Biotechnol 102: 47-90, 2006

29. Bendrea AD, Cianga L and Cianga I. Review paper: progress in the field of conducting polymers for tissue engineering applications. $\mathrm{J}$ Biomater Appl 26: 3-84, 2011

30. Richardson RR Jr, Miller JA and Reichert WM. Polyimides as biomaterials: preliminary biocompatibility testing. Biomaterials 14 : 627-635, 1993

31. Hirashima S, Ohta K, Hagihara M, Shimizu M, Kanazawa T and Nakamura K. Effect of surface texture of a polyimide porous membrane on the bone formation rate. J Hard Tissue Biol 27: 95100,2018

32. Khulbe KC, Feng CY and Matsuura T. Synthetic membranes for membrane process. In: Synthetic polymeric membranes: characterization by atomic force microscopy, ed by Pasch $\mathrm{H}$, Springer, Berlin, 6-9, 2008

33. Lonsdale HK. The growth of membrane techology. J Memb Sci 10: 81-181, 1982

34. Maniatopoulos C, Sodek J and Melcher AH. Bone formation in vitro by stromal cells obtained from bone marrow of young adult rats. Cell Tissue Res 254: 317-330, 1988

35. Hirashima S, Ohta K, Kanazawa T, Okayama S, Togo A, Uchimura N, Kusukawa J and Nakamura K. Three-dimensional ultrastructural analysis of cells in the periodontal ligament using focused ion beam/scanning electron microscope tomography. Sci Rep 6: 39435, 2016

36. Hirashima S, Ohta K, Kanazawa T, Uemura K, Togo A, Yoshitomi M, Okayama S, Kusukawa J and Nakamura K. Anchoring structure of the calvarial periosteum revealed by focused ion beam/scanning electron microscope tomography. Sci Rep 5: 17511, 2015

37. Ohta K, Sadayama S, Togo A, Higashi R, Tanoue R and Nakamura $\mathrm{K}$. Beam deceleration for block-face scanning electron microscopy of embedded biological tissue. Micron 43: 612-620, 2012

38. Mattioli-Belmonte M, Giavaresi G, Biagini G, Virgili L, Giacomini M, Fini M, Giantomassi F, Natali D, Torricelli P and Giardino R. Tailoring biomaterial compatibility: in vivo tissue response versus in vitro cell behavior. Int J Artif Organs 26: 1077-1085, 2003

39. Azad V, Breitbart E, Al-Zube L, Yeh S, O'Connor JP and Lin SS. rhBMP-2 enhances the bone healing response in a diabetic rat segmental defect model. J Orthop Trauma 23: 267-276, 2009

40. Van Vlierberghe S, Sirova M, Rossmann P, Thielecke H, Boterberg V, Rihova B, Schacht E and Dubruel P. Surface modification of polyimide sheets for regenerative medicine applications. Biomacromolecules 11: 2731-2739, 2010

41. Baek DH, Lee J, Byeon HJ, Choi H, Young Kim I, Lee KM, Jungho PJ, Pyo JD and Lee SH. A thin film polyimide mesh microelectrode for chronic epidural electrocorticography recording with enhanced contactability. J Neural Eng 11: 046023, 2014

42. Pirraco RP, Cerqueira MT, Reis RL and Marques AP. Fibroblasts regulate osteoblasts through gap junctional communication. Cytotherapy 14: 1276-1287, 2012

43. Pirraco RP, Marques AP and Reis RL. Cell interactions in bone tissue engineering. J Cell Mol Med 14: 93-102, 2010

44. Almubarak S, Nethercott H, Freeberg M, Beaudon C, Jha A, Jackson W, Marcucio R, Miclau T, Healy K and Bahney C. Tissue engineering strategies for promoting vascularized bone regeneration. Bone 83: 197-209, 2016

45. Zeidan AA, Radstrom P and van Niel EW. Stable coexistence of two Caldicellulosiruptor species in a de novo constructed hydrogen-producing co-culture. Microb Cell Fact 9: 102, 2010

46. Killian ML, Cavinatto L, Galatz LM and Thomopoulos S. Recent advances in shoulder research. Arthritis Res Ther 14: 214, 2012

47. Depres-Tremblay G, Chevrier A, Snow M, Hurtig MB, Rodeo $\mathrm{S}$ and Buschmann MD. Rotator cuff repair: a review of surgical techniques, animal models, and new technologies under 
J.Hard Tissue Biology Vol. 27(3): 185-194, 2018

development. J Shoulder Elbow Surg 25: 2078-2085, 2016

48. Needleman I, Tucker R, Giedrys-Leeper E and Worthington H.

Guided tissue regeneration for periodontal intrabony defects--a

Cochrane Systematic Review. Periodontol 2000 37: 106-123, 2005 